Clio. Femmes, Genre, Histoire

3| 1996

Métiers. Corporations. Syndicalisme

\title{
Les femmes dans les métiers parisiens : XIIIe-XVe siècles
}

\section{Simone ROUX}

\section{(2) OpenEdition \\ 1 Journals}

Édition électronique

URL : http://journals.openedition.org/clio/460

DOI : 10.4000/clio.460

ISSN : 1777-5299

Éditeur

Belin

Édition imprimée

Date de publication : 1 avril 1996

ISSN : 1252-7017

Référence électronique

Simone ROUX, "Les femmes dans les métiers parisiens : XIIle-XVe siècles », Clio. Histoire, femmes et sociétés [En ligne], 3 | 1996, mis en ligne le 01 janvier 2005, consulté le 19 avril 2019. URL : http://

journals.openedition.org/clio/460 ; DOI : 10.4000/clio.460

Ce document a été généré automatiquement le 19 avril 2019

Tous droits réservés 


\title{
Les femmes dans les métiers parisiens : XIIIe-XVe siècles
}

\author{
Simone ROUX
}

1 Le travail féminin dans l'atelier et la boutique, l'évolution éventuelle du rôle des femmes dans les activités urbaines, tels sont les points que ces quelques pages vont tenter d'éclairer. Pour ce faire, trois textes ou groupes de textes seront pris comme pivots de la réflexion: Le Livre des métiers d'Étienne Boileau prévôt de Paris, recueil daté ordinairement de $1268^{1}$, l'ordonnance royale de 1350 , le statut des lingères en $1485^{2}$.

2 En préalable, il convient de rappeler quelques données d'ensemble ${ }^{3}$. On sait que le travail des femmes est d'abord celui des paysannes, que les sociétés médiévales sont d'abord des sociétés rurales. En prenant l'exemple de Paris, ville exceptionnelle dans l'Occident, on choisit le cas extrême d'une société urbaine complexe et particulièrement développée ${ }^{4}$, à l'opposé de la situa-tion où vit la majorité des femmes de cette époque. Capitale du royaume, Paris est vue par les autorités royales comme un modèle pour les autres bonnes villes. On ne saurait donc hâtivement généraliser à partir du cas parisien. Mais son étude révèle jusqu'où, dans des conditions exceptionnelles, peut s'étendre le rôle des femmes dans la production et dans le monde du travail urbain médiéval. Ces quelques réflexions se situent d'emblée dans le champ de l'histoire sociale ${ }^{5}$.

3 Cette approche est fondamentale pour l'histoire des femmes, en général, et plus particulièrement pour la période médiévale car le devant de la scène documentaire y est accaparé par les écrits des clercs, des savants, des littérateurs de tous acabits qui répètent les mêmes discours sur l'infériorité des femmes, leurs faiblesses et leurs vices, le danger qu'elles font courir aux hommes qui ne se méfient jamais assez. On en tire de belles études sur la représentation de la femme dont on analyse l'impact sur toute la société. Mais on ne peut s'en tenir là. Aussi s'est-on intéressé à d'autres sources ${ }^{6}$ où l'on découvre des jeunes filles qui apprennent un métier, des femmes qui travaillent au même titre que les hommes pour gagner leur vie et celle de leurs enfants, des veuves qui gèrent le patrimoine familial ou l'atelier. Nous verrons surtout des sources normatives (statuts, ordonnances royales), mais les listes fiscales, les actes judiciaires, les ventes et les contrats de toutes sortes apportent aussi des informations concrètes et précises. Cet 
apport, ici simplement suggéré, est bien entendu un des fondements de toute étude sérieuse sur ce sujet.

4 L'idée première qui ordonne mon propos est simple, mais finalement j'ai choisi d'enfoncer cette porte prétendument ouverte. Le travail féminin, dans les villes médiévales, est une réalité indiscutable même s'il est difficile d'espérer en prendre l'exacte mesure chiffrée. Il a une place reconnue, légalisée dans des textes normatifs, il s'intègre légitimement dans les rouages de la société. Ce qui signifie que les femmes ne sont pas obligatoirement dans la dépendance matérielle de leur père ou de leur mari, qu'elles peuvent avoir des libertés d'action, qu'elles peuvent trouver dans le travail rémunéré non seulement des ressources, mais aussi une dignité, un rang, une reconnaissance de leur rôle dans le monde où elles vivent.

5 A cet égard, les statuts de métier sont essentiels. Dans ce qui va suivre, l'accent sera mis non sur cette donnée de base mais sur les formes, les limites, les changements éventuels qui peuvent marquer la réalisation concrète de cette possible liberté.

Le registre d'Étienne Boileau au XIIIe siècle

7 Le Livre des métiers est un recueil de plus de cent statuts de métiers parisiens, enregistrés au Châtelet devant le prévôt de Paris, Étienne Boileau. On pense que le recueil fut constitué en 1268 ; on sait ce que voulait le prévôt car il s'en explique dans l'introduction : faire écrire les règles observées par chaque métier, leur donner ainsi une force supplémentaire tout en les plaçant sous l'autorité royale qui garantit leur totale légitimité. Cent un métiers ont fait enregistrer leurs statuts. Étienne Boileau explique pourquoi il a voulu faire mettre par écrit d'abord ces règlements, puis la liste des tarifs de péage et autres taxes et enfin le texte des privilèges dont jouissent les seigneurs dans la ville de Paris. Ainsi dans toute la capitale, les activités et le commerce pourront être convenablement exercés sous l'autorité de l'officier royal qu'est le prévôt et du tribunal du Châtelet. Les manuscrits de ce recueil ne nous sont pas parvenus complets : manquent la troisième partie, et une bonne part de la seconde. Mais la première parait complète. Les statuts homologués sont de longueur variable : certains articles sont vraiment courts (une dizaine), d'autres assez détaillés (une quarantaine). Ils obéissent tous à une sorte de plan commun. Ils disent d'abord si le métier est franc ou s'il doit être «acheté » au roi ; ils fixent les règles de l'apprentissage - durée et conditions dans lesquelles le contrat est rompu ; ils évoquent ensuite les questions de qualité du travail - c'est là qu'on trouve des informations sur les techniques et les formes du travail ; ils passent ensuite aux jurés qui contrôlent le métier et indiquent les obligations et charges dont celle d'assurer le guet la nuit dans la ville.

$8 \quad$ Les textes résultent donc d'une volonté politique (celle du roi représenté directement par son prévôt) de policer la capitale, et du mouvement des métiers eux-mêmes qui viennent déclarer leurs statuts et qui obtiennent une reconnaissance de leur autonomie relative et de leurs droits. Par la suite, ces textes ont été complétés. D'autres métiers, à leur tour, ont fait enregistrer leurs statuts, soit qu'ils se scindent (ainsi les métiers du bois qui au XVe siècle se divisent entre charpentiers et menuisiers-ébénistes) soit qu'ils se dotent de statuts après avoir vécu sans communauté officiellement organisée et reconnue, soit que d'anciennes communautés estiment nécessaire de modifier ou de préciser le texte primitif. Ces documents reflètent ainsi l'évolution du travail urbain, couchent par écrit les problèmes soulevés par l'application des règlements et les réponses des métiers, fondées sur l'expérience et sur le point de vue des maîtres et jurés qui gouvernent la communauté. 
On a donc un ensemble de sources riches (une des bases pour atteindre l'histoire du travail) et délicates à interpréter. En effet, un statut dit l'idéal tracé, non la réalité exacte de sa mise en œuvre. Ensuite toutes les questions ne sont pas abordées dans ce genre de texte. Enfin, les métiers organisés n'encadrent pas la totalité des activités urbaines, on peut même dire qu'ils représentent une forme d'élite pourvue de privilèges et fonctionnant comme telle. On peut tirer beaucoup du recueil d'Étienne Boileau parce qu'il dresse un tableau de l'organisation des métiers à une période où la croissance parisienne est toujours vive, où se normalisent dans ces statuts les progrès et les innovations $d u$ siècle précédent. On y voit les métiers parisiens au temps de la prospérité et de l'optimisme.

Ce qui frappe d'abord est la présence de statuts de métiers entièrement féminins ${ }^{7}$. Ils concernent le travail de la soie. On ne sait comment, à Paris, les femmes ont ainsi monopolisé ces métiers de luxe. Lorsqu'ils apparaissent dans les statuts, ils se déclarent exactement comme les autres métiers parisiens. L'organisation interne, les conditions de l'apprentissage, le contrôle de la qualité des produits, l'intégration d'ouvrières étrangères, toutes ces exigences sont les mêmes que celles d'un métier masculin. Un seul article dans le statut des fileresses de soie à grands fuseaux, aborde une question plus particulière. Il concerne les plaintes de personnes qui ont confié de la soie à une fileuse laquelle ensuite nie l'avoir reçue, qu'elle l'ait vendue ou mise en gage. Évidemment cette forme d'escroquerie est punie : une amende de 5 sous, l'interdiction d'exercer le métier tant que la soie n'a pas été rendue et le dommage causé réparé. Mais faut-il voir dans la mention précise de ce type de vol une conséquence des vices et des faiblesses attribués aux femmes? C'est ce que pense A. Franklin, historien du début du siècle qui en citant ce statut dans son dictionnaire ${ }^{8}$ croit suffisant de dire comme une évidence que les fileresses de soie étaient les plus débauchées des ouvrières parisiennes, ce qui expliquerait, selon lui, qu'elles soient les seules à avoir fait préciser que les contrats d'apprentissage devaient être écrits. On saisit mal le raisonnement qui lie débauche et contrat écrit mais on y lit la dépréciation systématique du travail féminin. L'historien actuel se méfie de ces jugements et il cherche la raison de cet article plutôt dans la grande valeur du matériau traité et dans la tentation de s'en emparer à bon compte qui peut saisir aussi bien les hommes que les femmes. D'autant plus que, dans ce genre de conflit, la mauvaise foi n'est pas nécessairement du côté des fileuses ${ }^{9}$.

11 Dans beaucoup de métiers, la main-d'œuvre féminine est explicitement mentionnée ${ }^{10}$. Hommes et femmes travaillent ensemble. Les textes de statuts écrivent «nul ou nulle ne peut... », parlent d'apprentis et d'apprenties, de maîtres et de maîtresses, de "preud'homes » et de "preude fames ». Le témoignage du vocabulaire est décisif: il enregistre la réalité du travail féminin accepté et intégré à tous les échelons de la hiérarchie interne du métier. Mais il est difficile de dépasser ce constat car les statuts ne décrivent pas la répartition des tâches à l'intérieur du métier ${ }^{11}$.

Ils sont beaucoup plus diserts sur les femmes de maitres ou de compagnons. L'accouchement de l'épouse est une excuse pour dispenser le mari du service de guet la nuit, au même titre que sa propre maladie ou sa vieillesse ${ }^{12}$. Dans le statut des fripiers on relève un article plus détaillé sur ce point. Profitant de la déclaration officielle de leurs statuts, les fripiers y ajoutent une plainte et une demande dont on ne sait si elle a abouti. Ils disent que les gens du roi ne leur permettent plus, lorsqu'ils sont malades, de se faire remplacer par un serviteur ou un voisin, mais « ils font venir leurs femmes... soit belles soit laides, soit vieilles soit jeunes, soit faibles soit grosses, pour leurs maris excuser, 
laquelle chose est très laide et vilaine que une femme aille et reste au Châtelet depuis le couvre-feu aussi longtemps que le guet est effectué,... et qui s'en vont à cette heure parmi une ville comme Paris toute seules dans des rues éloignées de leurs hôtels, et cela a entrâné du mal, des péchés et des vilenies $»^{13}$.

A. Franklin juge le propos «naïf et pittoresque». L'historien d'aujourd'hui y lit une brimade envers les fripiers, métier mal considéré socialement. Le lien bien connu entre pauvreté-mépris-peur fonctionne à merveille. Le métier regroupe des fripiers travaillant en boutique, mais aussi des fripiers ambulants dont beaucoup étaient très pauvres. Il a mauvaise réputation: les fripiers sont suspectés de vendre trop cher, d'écouler des marchandises volées, d'être des receleurs en liaison avec les criminels. Les femmes des fripiers subissent de plein fouet la déconsidération qui pèse sur métier par cette obligation de remplacement. Les épouses de ces gens douteux non seulement n'ont pas à être protégées, mais encore on se demande si, en les faisant venir au Châtelet, il ne s'agit pas implicitement de signifier qu'elles sont là pour divertir les hommes du guet. Il est malaisé de saisir tout ce qui se cache derrière cette brimade, de faire la part de la réalité et des préjugés dans cette déconsidération qui blesse les fripiers. Mais il est sûr qu'en 1268 , le métier veut corriger cette image négative. En faisant homologuer ses statuts il obtient d'être considéré comme les autres métiers et il demande la suppression de cette contrainte pour le guet qu'il juge humiliante et dangereuse.

Ces textes posent presque tous le problème des veuves de maître et précisent dans quelles conditions elles peuvent poursuivre l'activité de leur mari défunt. Le plus souvent, si elles ne se remarient pas et qu'elles prennent à leur service un homme du métier (au cas où elles n'auraient pas elles-mêmes la compétence requise), ou si elles se remarient avec un homme du métier, elles peuvent travailler et gérer l'atelier. L'esprit de ces dispositions est clair. Les femmes voient leurs droits protégés, dans la mesure où ceux-ci n'affaiblissent pas le métier. Le risque n'est pas de laisser une femme diriger, il est d'introduire dans la communauté un second mari qui n'aurait pas les compétences exigées par les statuts ${ }^{14}$. Le travail féminin est placé devant les mêmes obligations que le travail masculin.

Deux métiers toutefois témoignent d'une défiance explicite envers le travail des femmes. Les corroiers ${ }^{15} c^{\prime}$ est-à-dire des fabricants de courroies et de ceintures précisent qu'une fille de corroier qui a terminé son apprentissage ne peut s'installer en ville que si elle se marie avec un corroier. Et d'expliquer cette restriction ainsi : les filles qui s'établissaient à leur compte et prenaient des apprentis «ne faisaient que tromperie et ribauderie », elles gaspillaient l'argent que leur avait donné leurs parents pour s'établir (le texte est fort et dit «emblé » c'est-à-dire volé), et quand elles étaient sans ressources, elles revenaient chez leurs parents qui ne pouvaient pas, sans plus grand péché encore, ne pas les reprendre chez eux. A l'évidence, cette explication inscrite dans les statuts traduit un préjugé largement répandu qui veut que les filles plus faibles et tentatrices soient plus sensibles à l'attrait du péché et de la chair. En les obligeant à épouser un homme du métier, les corroiers estimaient discipliner et surveiller leurs filles. Je rapprocherai toutefois cette défiance de celle que suscitent tous les jeunes, garçons ou filles, et que plusieurs statuts évoquent clairement lorsqu'ils parlent de l'apprentissage. Le maître doit exercer sur eux une forte autorité, certes paternelle, mais rigoureuse. On peut ajouter une autre raison possible à cette obligation faite aux filles de corroier : mieux contrôler l'expansion du métier, mieux se répartir la clientèle, soit la tendance bien connue des communautés de métiers à limiter la concurrence. 
16 Autre indice d'une défiance envers le travail des femmes, un article des statuts des cristalliers. Ceux-ci expliquent que la veuve d'un maître peut continuer à exercer (dans les conditions habituellement définies) mais qu'elle ne peut prendre d'apprentis, car le métier est trop «soutil», entendons qu'il requiert une grande habileté et intelligence. Une femme est capable de travailler le cristal de roche et les pierres semi-précieuses mais elle ne peut pas transmettre son savoir à un enfant. Est-ce à dire que les maîtres craignent que, sous son autorité, les apprentis ne gâchent un matériau cher ? Pensent-ils que les femmes peuvent exécuter mais ne peuvent dominer l'art et la technique suffisamment pour pouvoir enseigner correctement?

Les statuts du XIIIe siècle montrent les femmes regroupées en communautés féminines ou travaillant avec des hommes, dans les métiers du textile, dans ceux du vêtement et de la parure, mais pas dans le bâtiment, ni dans les métiers du bois ou des métaux. Dans ceux de l'alimentation, il est assuré qu'elles s'occupent surtout de la boutique, du contact avec la clientèle. Lorsqu'on cherche à dresser une liste des métiers féminins, y compris ceux qui ne sont pas organisés en communautés, on constate qu'elles gagnent leur vie souvent dans le tout petit commerce de détail.

18 Toute une gamme de rapports au travail se dessine donc qui offre aux femmes la possibilité d'une autonomie réelle ${ }^{16}$. Mais à revenu équivalent, c'est l'intégration professionnelle dans une communauté de métier qui est la plus sûre. L'endogamie des gens de métier, encouragée par les statuts, favorise les filles de maître, qu'elles soient apprenties dispensées de chef d'œuvre ou épousées par le valet/compagnon qui ainsi peut mieux s'établir. Les statuts se fondent aussi sur la collaboration attendue de la femme du maître, souvent issue du milieu du métier, qui, sans avoir appris dans les règles, s'y connaît assez pour aider à la gestion, et pouvoir, en cas de veuvage continuer les activités. Le recueil de 1268 nous révèle un monde artisanal bien ordonné, ménageant pour les femmes des espaces d'initiative et d'autonomie matérielle.

L'ordonnance de $1350^{17}$

Un siècle après, le climat d'ensemble a bien changé. La Grande Peste de 1348 vient de ravager la capitale, alors que l'économie est entrée dans une phase de longue dépression et que la guerre étrangère ébranle les fondements de l'ordre ancien. Paris a réussi à absorber ces chocs, mais le royaume est atteint. L'autorité royale estime nécessaire d'intervenir directement dans le jeu social qu'elle estime dévoyé. Paris doit donner l'exemple de la remise au travail.

21 L'ordonnance de 1350, publiée l'année suivante, analyse le mal et fixe les remèdes. Le mal provient de ce que la main d'œuvre, raréfiée après l'épidémie, se fait plus exigeante et que les donneurs d'ouvrage cèdent et accordent des hausses de salaires jugées excessives. L'ordonnance prise au nom de Jean le Bon dénonce cet état de fait. Alors que les bras manquent pour faire l'ouvrage, Paris voit des gens « oyseux » qui préfèrent «truander » plutôt que "d'exposer leur corps à faire aucune besogne", qui passent leur temps à la taverne et au bordel. Et le reproche vise " tant les hommes que les femmes"

22 L'analyse moralisante se fonde sur une réalité. Dans un rapport de forces qui leur est momentanément favorable, les salariés savent faire pression, refusent de travailler aux anciens tarifs et, en attendant, " profitent de la vie » après la terrible ponction de la mort noire.

23 Le roi prend des mesures extraordinaires. Par-dessus les privilèges et statuts de métiers, il en ouvre largement l'accès à tous ceux, Parisiens ou non, hommes ou femmes, qui 
veulent s'établir et travailler dans la ville. Surtout l'ordonnance entre dans une énumération des métiers pour fixer le salaire maximum qui peut être payé, à savoir celui d'avant la peste, et prévoit des sanctions contre les salariés et les employeurs qui ne respecteraient pas l'ordonnance et ses tarifs. Inutile d'ajouter que l'application de ces mesures a été quasi impossible.

J'ai choisi d'évoquer ce texte fort célèbre pour voir comment le travail féminin y est représenté. Il donne un panorama plus étendu que celui des statuts. Ainsi sous la rubrique "salères des femmes » il est dit que toutes les femmes qui s'embauchent pour servir ne peuvent avoir plus de douze deniers par jour si c'est tout leur salaire, et six seulement si elles sont logées ou nourries. Sont concernées par l'article toutes les servantes, à la tâche ou à la journée ou à l'année, qui travaillent chez les particuliers.

Cet emploi féminin est organisé par des femmes qui tiennent des sortes de bureau de placement. On les nomme commanderesses ou recommanderesses ${ }^{18}$. Elles placent des nourrices et des chambrières, professions féminines qui n'ont ni communauté de métier ni statuts mais qui existent depuis longtemps. L'ordonnance royale ne les oublie pas et fixe le nombre de placements que les commanderesses peuvent faire et le tarif de leurs salaires, soit douze deniers pour une chambrière et deux sous pour une nourrice, gages comprenant ce qui est payé par l'employée et par l'employeur. Les chambrières ne doivent percevoir que trente sous par an au maximum, et on peut en déduire ce qui leur est payé en nourriture ou vêtement. Les nourrices ont de la même manière cinquante sous par an et pas plus. L'ordonnance leur impose de terminer le temps de leur contrat, sans doute parce que certaines chambrières ou nourrices ont cherché une autre place mieux payée. Il est certain qu'avec ces métiers de service on touche là une large part de la main-d'œuvre féminine à Paris ${ }^{19}$.

Autre travail qui n'a pas donné lieu à la constitution d'une communauté de métier : les lavandières dont le salaire, payé à la pièce de linge lavé, est également fixé à un denier. De même les femmes qui travaillent aux vignes, celles qui lavent la panse des pourceaux et celles qui font les andouilles et les boudins, voient leurs salaires définis dans l'ordonnance royale. Car à la différence du recueil de statuts, ce texte part de la réalité concrète, des postes de travail ou des emplois divers exercés par des femmes, que cela corresponde ou non à un métier réglementé.

En tant que main-d'œuvre comprise dans la communauté d'un métier, les femmes, dans l'ordonnance, n'ont pas à être distinguées des hommes; leur sont appliquées les mêmes mesures d'ouverture et d'accueil, car une ouvrière qualifiée était aussi recherchée qu'un homme. Les pingeresses (peigneuses) de laine de soie ou de chanvre, ont un article spécial dans l'ordonnance alors qu'elles font partie du métier des cardeurs, sans doute parce que, au milieu du XIVe siècle, elles commencent à s'organiser à part à l'intérieur de ce métier ${ }^{20}$

L'ordonnance apporte donc des compléments d'informations importants. Elle montre, tant pour les femmes que pour les hommes d'ailleurs, que le monde des métiers réglementés est loin d'encadrer tout le travail urbain. Il conviendrait d'élargir encore le champ de l'enquête. Je ne fais ici que signaler des pistes. Dans les métiers relevant de la santé et des soins du corps, il y a des femmes : des « miresses ", des " herbières ", et " des ventrières » : entendons des femmes qui pratiquent une médecine empirique (méprisée par les physiciens, médecins savants), des femmes qui vendent des herbes médicinales (et sans doute des conseils de santé), des sages-femmes. Mais on trouve encore des barbières 
(méprisées comme les barbiers par les chirurgiens, eux-mêmes méprisés par les médecins de longue robe), des femmes qui tiennent des étuves et des «laveuses de tête ».

Les professions artistiques comprennent des femmes, «jongleresses », «menestrelles », musiciennes, danseuses. Elles partagent avec les hommes une forme de déconsidération que les ménestrels, par exemple, ont tenté de faire disparaître en s'organisant en communauté de métier ${ }^{21}$. Il y avait des femmes peintres, sculpteurs, sans doute travaillant dans l'équipe de l'atelier sous la direction du maître. Enfin, on sait que les sources fiscales mentionnent quelques « mestresses d'école » à qui était confiée l'éducation primaire des petites filles, sous l'autorité de l'évêque et des curés.

On peut donc estimer qu'à Paris, les femmes avaient de grandes possibilités d'assurer leur autonomie matérielle en travaillant. Les seules professions qui les excluaient radicalement étaient celles qui supposaient des études universitaires, même rudimentaires, puisque l'accès aux facultés était réservé aux clercs et que l'Église interdisait la cléricature aux femmes. Les professions judiciaires, les charges et offices de tous niveaux leur étaient fermés. Elles pouvaient sans doute gagner leur vie par leur travail, peut-être acquérir aisance et respectabilité, mais elles ne pouvaient songer à de belles carrières ouvrant vers les pouvoirs et le commandement. Le statut des lingères en $1485^{22}$

32 A la fin du XVe siècle, la situation d'ensemble est globalement meilleure. La capitale a retrouvé dynamisme et relative prospérité. Mais Paris a connu, dans la première moitié $\mathrm{du}$ siècle, une période très noire : la guerre étrangère et civile, la crise politique (liée notamment à la domination anglo-bourguignonne de 1418 à 1435), la dépopulation et l'appauvrissement, tout s'était conjugué pour ruiner la ville. Elle ne commence à se remettre que dans la seconde moitié $\mathrm{du} \mathrm{XVe}$, et ces expériences douloureuses ont beaucoup marqué les esprits. On aspire au retour à la paix, au respect de l'ordre ancien, ce qui n'est pas nécessairement favorable aux femmes.

Mais en même temps, la grande ville reprenant son expansion, les possibilités concrètes de travailler et de se garantir une autonomie matérielle augmentent et se diversifient.

On a ainsi le sentiment que sont à l'œuvre deux tendances contraires. L'une de retour à l'ordre qui veut imposer le modèle de l'épouse vivant dans sa maison, comme les femmes de bons bourgeois et de nobles, fières de ne pas être contraintes de travailler, et l'autre qui, s'appuyant sur l'essor des activités de la grande ville, pousse à affiner les statuts, enregistre les changements et les nouveaux métiers, car dans la masse de la population, il $\mathrm{y}$ a des Parisiennes qui ont besoin de pourvoir à leur subsistance et pour qui un métier, surtout s'il est organisé, offre tout à la fois des ressources, la sécurité et la solidarité du groupe $^{23}$. Un examen de l'ensemble des sources normatives que sont les statuts montrerait sans doute que les femmes continuent à y tenir leur place, voire qu'elles l'augmentent. Et cela se poursuit jusqu'au XVIIIe siècle.

35 En choisissant le statut des lingères octroyé par le roi en 1485 , je veux évoquer la première tendance, celle qui viserait plutôt à restreindre l'espace de liberté des femmes au travail sur qui pèse le soupçon systématique de débauche et de scandale visant à la déconsidération sociale. Retour à l'ordre et mise au pas s'imbriquent dans l'histoire sociale et politique de Paris du second XVe siècle.

Résumons d'abord ce texte. Les maîtresses du métier ne se réclament pas d'un statut antérieur qu'il faudrait confirmer, mais d'un privilège octroyé par saint Louis. En effet, il est attesté que ce roi a permis aux pauvres lingères de Paris d'établir leurs étals près du 
cimetière des Innocents, face aux murs des Halles. Elles pouvaient y vendre le produit de leurs travaux sans payer de taxes. Le texte du privilège, précisé, confirmé, a toutefois été perdu et il faut en refaire un nouveau. Le métier en a besoin, parce que le retour à l'ordre est nécessaire (le thème est courant à l'époque, où l'on répare ce que les malheurs du temps ont détruit ou fait oublier) et surtout, ce qui est plus intéressant, parce que le métier accueille des filles de notables et bourgeois, de gens de justice, que leurs parents placent là pour leur faire apprendre l'» œuvre de couture » et » honnête maintien » et pour « éviter l'oiseveté ». Dans ces conditions, le métier ne peut plus tolérer la présence de lingères «mal renommées » qui « se gouvernent mal ».

Le premier article du statut, après ces considérations, exclut donc des assemblées et fêtes du métier qui se tiennent chaque année, ces lingères «blâmées et scandalisées de leurs corps ou autrement». Les autres articles précisent que les maitresses du métier convoqueront les lingères dont la conduite leur déplait, et si celles-ci ne s'amendent pas, elles seront exclues et leurs marchandises ne pourront être mises près des Halles comme le permet le vieux privilège. Si elles ne veulent pas obéir aux maîtresses et prétendent continuer à venir aux assemblées et étaler aux Halles, il sera fait appel à la force publique (officiers et sergents du Châtelet) pour les faire partir.

L'interprétation de ce règlement pose bien des questions. D'abord, on ne peut prendre pour l'exacte et entière vérité les accusations de débauche et de crime qui accablent tellement de lingères que l'exclusion apparaît comme l'axe central du règlement. Certes, dans beaucoup de statuts on trouve quelques phrases qui rappellent que les gens du métier, leurs femmes et leurs familles, doivent jouir d'une bonne réputation, avoir une bonne moralité, mais ce n'est jamais comme ici le thème unique.

Que la prostitution, liée à l'insuffisance des ressources, se soit développée avec les crises est certain ${ }^{24}$, ce qui a sans doute alimenté une constante suspicion à l'égard du travail des femmes. L'atelier et la boutique tenus par des femmes serviraient de façade à un commerce moins honorable ${ }^{25}$. C'est ainsi que Franklin, à l'article lingère de son dictionnaire, adopte ce point de vue sans aucune réticence. Il cite à l'appui Savary, auteur d'un dictionnaire du commerce et des métiers publié en 1728 , qui présente le privilège donné par saint Louis comme un moyen de restreindre la prostitution, et ajoute que le saint roi en fut pour sa peine. On pourrait trouver d'autres traces écrites de ce préjugé calomnieux qui affleure vite quand on parle du travail des femmes. L'histoire de Jeanne la Quentine, rapportée dans Le Mesnagier de Paris, est révélatrice ${ }^{26}$. Cette épouse d'un bon notable parisien apprend que son mari a une liaison avec une jeune lingère très pauvre. Elle ne dit rien à l'époux coupable ; mieux, elle va trouver la lingère, lui explique que son mari est habitué à plus de confort et elle équipe le logement de la fille pauvre : lit douillet, vêtements, provisions... Et d'ajouter que cela doit rester un secret entre elles. Quand le mari retourne voir la lingère il est stupéfait de la trouver si bien installée et n'hésite pas sur l'explication: sa belle est tombée dans la prostitution et il entre dans une colère si violente que la lingère finit par lui dire la vérité. Repentant, le mari rentre au foyer, ne dit rien et sa femme non plus. Morale expliquée de l'histoire : l'épouse n'a pas à juger les fautes de son mari, mais, par sa discrétion, sa patience, elle peut l'aider à sortir du péché et retrouver l'harmonie dans son ménage. Dans l'histoire, la lingère a pu garder les draps de lit, les couvertures et les provisions que l'épouse lui avait fait porter. Autant que la portée supposée de cette morale, la vision du travail féminin est intéressante : une lingère honnête ne peut qu'être très pauvre. Un surcroît de ressources ne peut être que le fruit d'un commerce honteux et condamnable. 
40 Revenons à nos lingères «mal renommées ou scandaleuses » dont il faut débarrasser le métier. En utilisant contre elles l'argument moral, on peut estimer que les maitresses, plus profondément, leur adressent un reproche général : elles font partie du monde des gens de " petit état ", gens " méchaniques », qu'il faut remettre à leur place, c'est-à-dire une place humble qui ne leur laisse que le devoir d'obéissance. N'ont-ils pas essayé, lors des troubles et révoltes urbaines, de jouer un rôle, d'intervenir dans la politique et le gouvernement de la ville, chose scandaleuse comme le disent les autorités ${ }^{27}$ ? Peut-on laisser ces petites ouvrières pauvres côtoyer, dans la même communauté, les jeunes demoiselles des familles notables de Paris? Les maîtresses du métier, sensibles à la pression sociale qui les pousse à cette sévérité moralisante, n'ignorent tout de même pas que l'accusation de débauche et de mauvaise renommée peut s'étendre abusivement. L'article cinq du statut le montre. Il prévoit que les lingères exclues, si elles ont fait leur apprentissage, pourront tenir en ville des ouvroirs, travailler normalement, à condition de ne plus paraître ni aux Halles, ni dans les assemblées et fêtes. Est-ce trop solliciter le texte que de chercher une sorte de ségrégation sociale ${ }^{28}$ dans ces mesures réclamées par des femmes contre d'autres femmes pourtant du même métier?

On le voit, il est malaisé de donner, en l'état actuel, des conclusions trop péremptoires. Nous avons encore beaucoup à apprendre et donc à chercher sur l'histoire des femmes au Moyen Age. Les sources qui semblent les plus simples et directes comme le sont les statuts de métier, requièrent une attention plus exigeante que celle qui leur est généralement accordée. Elles doivent être examinées dans leur ensemble et non seulement pour y chercher quelques confirmations d'idées a priori. Ces quelques pages ont tenté de montrer la complexité de l'analyse, mais ce n'est qu'une ébauche. Il faudrait, plus rigoureusement, reprendre tous les textes et les comparer à d'autres sources. Pour le moment, je crois difficile de trancher sur le sens de l'évolution d'ensemble. Au cours de ces trois siècles, les femmes dans les métiers organisés et plus généralement dans les activités urbaines ontelles vu leur rôle et leur place croître, entraînant une amélioration de leur condition sociale ? Est-il juste d'ailleurs de se borner à marquer d'un signe plus ou d'un signe moins un mouvement qu'on connaît mal encore mais dont on sait cependant qu'il ne fut sans doute ni linéaire ni simple?

Cette prudence ne doit cependant pas amener à oublier les acquis des recherches. Il est peu probable que les études à venir sur Paris viennent contredire l'hypothèse selon laquelle les sociétés médiévales, et notamment les sociétés urbaines, ont su ménager ou ont dû accepter (après quelle luttes et quelles résistances?) un espace de liberté par le travail pour les femmes, et que en phase d'économie dynamique (comme au XIIIe siècle), ces possibilités se concrétisent plus facilement et plus largement qu'en période de crise ou de récession.

Reste à exploiter ce champ d'enquête maintenant bien cerné qui associe étroitement histoire des femmes et histoire sociale. S'y lancer suppose un travail long et dans le cas de Paris, plutôt ingrat ${ }^{29}$. Mais on ne peut en faire l'économie. Tout ce qui sera fait, à condition de ne pas tenter de conclure prématurément, apportera du solide ${ }^{30}$.

$C^{\prime}$ est bien ce que montre à sa manière le dictionnaire d'A. Franklin. Le travail et les métiers féminin apparaissent dans les articles, référencés avec soin, parfois expliqués. Un des intérêts de l'ouvrage tient à ce qu'il suit, autant que possible, l'évolution jusqu'au XVIIIe siècle. Ces apports conservent leur valeur et la réimpression du dictionnaire indique bien qu'aucune recherche n'a pour le moment remplacé cette synthèse. On aimerait être assuré que les recherches actuelles conserveront, après un siècle, autant 
d'intérêts pour les futurs historiens. L'ouvrage comprend aussi un index/table des matières, développé et fort utile, car un lecteur peut avoir besoin d'un ordre plus synthétique que l'ordre alphabétique. Or, en y cherchant ce qui concerne le travail féminin on tombe au mot femmes, sur les renvois suivants: " -femmes dentistes, femmes fortes, hercules de foire, - imitent le costume des hommes, - maîtresse au logis, fines tailles des femmes, - n'allaient point au théâtre ». Il serait facile d'ironiser. Il est plus fructueux de souligner combien cette courte liste de renvois (qui mériterait un commentaire que je laisse aux spécialistes de l'histoire du XIXe siècle) est en contradiction avec toute la démarche historique de l'auteur puisque celui-ci a bien traité de ce sujet, le travail féminin, mais qu'il ne l'a pas identifié lorsqu'il a constitué l'index.

Ce qui rassure, à la lumière de cet exemple, est que les recherches en cours ne risquent plus de négliger, dans l'histoire sociale de la capitale, la dimension importante du travail féminin. On peut espérer qu'elles sauront aussi éviter le piège des préjugés et des schémas implicites et caricaturaux quand viendra le temps des nouvelles synthèses qu'elles travaillent à préparer.

\section{BIBLIOGRAPHIE}

CAZELLES, Raymond

1972 Paris, de la fin du règne de Philippe Auguste à la fin du règne de Charles V, 1223-1380, Paris, Hachette, « Nouvelle Histoire de Paris ».

CHEVALIER, Bernard

1982 « Corporations, conflits politiques et paix sociale en France aux XIVe et XVe siècles ", Revue Historique, $543: 18$ à 44 .

DUBY Georges et PERROT Michelle (dir.)

1991 Histoire des femmes en Occident, tome 2, Le Moyen Age, sous la direction de

C. Klapisch-Zuber, Paris, Plon.

ENNEN, Edith

1984 Frauen in Mittlelater, Müchen C.H. Beck [trad. angl. The medieval woman, Oxford, Basil Blackwell, 1989].

FAVIER, Jean

1974 Paris au XVe siècle, Paris, Hachette « Nouvelle Histoire de Paris ».

FRANKLIN, Alfred

1906 Dictionnaire historique des arts, métiers et professions exercés dans Paris depuis le XIIIe siècle, Paris [réédité par Laffite Reprint, Marseille, 1987].

GEREMEK, Bronislaw

1976 Les Marginaux parisiens aux XIVe et XVe siècles, Paris, Flammarion. 
GRIMAL Pierre (dir.)

1966 Histoire mondiale de la femme, Paris, Nouvelle Librairie de France, tome II.

LESPINASSE, René de

1879 Les Métiers et corporations de la ville de Paris : le livre d'Étienne Boileau, Paris, Histoire générale de Paris.

LESPINASSE, René de et BONNARDOT, François

1886-1897 Les Métiers et corporations de la ville de Paris. Recueil, statuts règlements depuis le XIVe siècle jusqu'à la fin du XVIIIe siècle , 3 vol. , Paris, Histoire générale de Paris.

Le Mesnagier de Paris,

1994 édition par Georgina E. Brereton et Janet M. Ferrier, traduction et notes par Karin Ueltschi, Paris, Livre de Poche, coll. « Lettres Gothiques ».

POWER, Eileen

1976 Medieval Women, Cambridge University Press, [trad. fr.. Les Femmes au Moyen Age, Paris, Aubier Montaigne, 1979].

ROSSIAUD, Jacques

1988 La Prostitution médiévale, Paris, Flammarion

ROUX, Simone

1994 Le monde des villes au Moyen Age, XIe-XVe siècle, Paris, Hachette, « Carré Histoire ».

\section{NOTES}

1. De Lespinasse 1879.

2. De Lespinasse et Bonnardot, 3 vol.1886, 1892, 1897. Les citations des textes originaux en vieux français ont été modernisées.

3. Grimal 1966 et Duby et Perrot 1991.

4. Pour une vue d'ensemble, se reporter à Cazelles 1972, et Favier 1974. La population parisienne, au cours de la période, a atteint plus de 200000 âmes et lors de la grande dépression de la ville, dans la première moitié du XVe siècle, on estime à 1000000 la population restante ; ensuite la croissance reprend et à la fin du XVe siècle, les hauts chiffres du début du XIVe sont dépassés.

5. Roux 1994, pour des données d'ensemble.

6. Ces sources, plutôt avares en discours explicatifs apportent des informations directes, peu nombreuses (à notre goût), d'interprétation parfois délicate. Mais elles seules permettent de fonder une recherche solide sur l'histoire sociale des femmes.

7. Il s'agit des fileresses de soie à grands fuseaux, des fileresses de soie à petits fuseaux, des tisserandes de soie, des tisserandes de couvre-chefs en soie, des fesseresses de chapeaux et « d'œuvres à quatre pertuis ». Donc une extrême spécialisation qu'on retrouve dans toute l'organisation du travail à Paris. On note que si l'essentiel du gouvernement interne de la communauté est bien dans les mains des femmes, des hommes sont mentionnés : les deux statuts des fileuses indiquent que le métier est sous l'autorité de deux jurés nommés par le roi. Mais les tisserandes de soie sont surveillées par trois maîtres et trois maîtresses jurés, également nommés par le roi. 
8. A. Franklin 1906 très utile par la quantité et la qualité des informations qu'il contient. Mais il est aussi intéressant par les appréciations qu'il donne sur le travail, les ouvriers et sur la manière d'aborder cette histoire par les historiens du début du siècle. A. Franklin se contente de reprendre l'opinion de Lespinasse qui, dans les notes de son édition, insiste sur cet aspect.

9. Au XIIIe siècle, le donneur d'ouvrage Jean Boinebroke, marchand et échevin de Douai usait de ces procédés pour sous-payer le travail des femmes notamment. On sait qu'il avait demandé à ses exécuteurs testamentaires de réparer ses fautes et ceux-ci ont recueilli les plaintes des ouvriers et ouvrières qui racontent comment, par exemple, Boinebroke fournissait des sacs de laine dont seul le dessus était de bonne qualité, et ensuite accusait ses ouvrières d'avoir triché sur la marchandise travaillée ce qui lui permettait de diminuer leurs salaires. Voir G. Espinas 1933.

10. Vingt métiers sur les cent un enregistrés présentent ce caractère mixte, que ce soit par une seule référence ou par plusieurs articles qui évoquent le travail des femmes. 11. Chez les «braeliers de fil » entendez les fabricants de braies, sorte de pantalon, un article est consacré à l'apprentissage pour les femmes ; il est d'une durée de deux années alors que dans le reste du métier il est de sept ans, sans doute parce qu'il s'agit d'une tâche subordonnée dans la fabrication, le statut indique " pour coudre et apprêter audit métier ». Cf. le titre XXXIX du Livre des métiers. On voit aussi, dans un article du statut des foulons (titre LIII) que les femmes ne peuvent intervenir dans le travail (« mettre la main au drap ») tant que le drap n'est pas tondu. Est-ce parce que les travaux de foulage des draps sont trop pénibles?

12. Trente-deux métiers font inscrire cette clause. Le guet, en 1268, semble être une lourde charge. Il n'est pas évoqué dans les statuts de métier féminin. On voit mal un règlement confirmé par l'autorité royale qui donnerait aux femmes un quelconque rôle dans le service des armes. On touche là à un secteur strictement réservé aux hommes dans la société médiévale.

13. Cf. le titre LXXVI du Livre des métiers.

14. Les fabricants de lacets de soie et de fil expliquent ainsi qu'un maître, célibataire ou marié à une femme qui n'est pas du métier, ne peut prendre qu'un apprenti, mais si sa femme est du métier, le couple peut en prendre deux. Même disposition chez les crépiniers et crépinières (passementiers). On le vérifie encore dans le statut des rubaniers, modifié en 1400 . Ce métier mixte comprend des maîtres et des maîtresses. Il y est précisé qu'à la mort d'une maîtresse, le mari veuf ne peut continuer à exercer que s'il est du métier, ou s'il se remarie avec une femme " qui en soit ouvrière ».

15. Cf. le titre LXXXVII du Livre des métiers.

16. Le droit canon et le droit civil permettent la séparation des époux sans que le mariage soit rompu. Les actes de la pratique témoignent que cette possibilité peut être réalisée : on trouve, en petit nombre il est vrai, des femmes séparées qui achètent ou vendent des biens et qui pour ce faire, n'ont plus besoin de l'autorisation de leur mari. Indications à rapprocher de celles des listes fiscales indiquant des feux féminins qui ne sont pas toujours ceux de veuves.

17. De Lespinasse 1879 tome $1: 25$ et suiv.

18. Rive droite, près des Halles une rue des Recommanderesses témoigne de l'importance de ce métier : c'était là, sans doute, l'emplacement principal de cette activité.

19. C'est ce que suggèrent aussi les sources fiscales où l'on peut relever les mentions de métier féminin. La quête sur ce point est assez décevante, mais dans les rares mentions, celle des chambrières est une des plus fréquentes. 
20. Il conviendrait de reprendre l'ensemble des statuts et des ordonnances pour voir les nouveaux métiers féminins qui s'organisent. Un exemple, en 1300 les femmes qui font les « aumosnières sarrazinoises » (à la mode orientale) font homologuer leur statuts de 14 articles : cf. Lespinasse 1886-1897 tome II : 13 et suiv.

21. De Lespinasse 1897 tome III : 580-582. Le statut de 1321 est juré par trente-huit personnes dont huit femmes.

22. De Lespinasse 1897 tome III : 69-70.

23. Il convient de dire que la communauté de métier se double très souvent d'une confrérie où les apprentis, les épouses, les servantes et serviteurs, c'est-à-dire toute la maisonnée qui vit au rythme de l'atelier ou de la boutique, se retrouve.

24. Les recherches sur la prostitution médiévale ont été renouvelées par les travaux de Rossiaud 1988. Mais les résultats n'en sont pas immédiatement transposables pour Paris, ville septentrionale qui n'a pas intégré, comme les villes méridionales, la prostitution dans ses institutions urbaines.

25. Cette suspicion semble fondée quand on considère les ordonnances royales et municipales. Geremek $1976: 264$, n. 41 qui cite un texte de 1420 où il est dit que les maquerelles « tiennent... des échoppes... de denrées et de métier », ce qui en, fait, leur permet d'accueillir jour et nuit une clientèle spéciale. De même, les recherches fondées sur les sources judiciaires donnent beaucoup d'exemples de femmes ouvrières criminelles et/ou prostituées. Toute la question est de savoir ce que représente, dans l'ensemble d'un métier ou d'un groupe social, cette population féminine délinquante. On ne peut adopter, sans précautions critiques, ni le point de vue des autorités de la ville, ni celui des officiers du roi.

26. Le Mesnagier de Paris 1994 : 402 à 407. Ce traité de morale et d'économie domestique, destiné à l'épouse toute jeune d'un bon notable parisien, date de la fin du XIVe siècle. 27. Chevalier 1982 analyse l'évolution de l'organisation des métiers en fonction de leur participation aux affaires publiques et municipales. Il fait remarquer combien la recherche de la considération est importante dans les conflits et dans les revendications des gens de métier.

28. Dans la période de restauration puis de retour à la croissance qui correspond à la seconde moitié du siècle, on peut trouver des indices exprimés dans des textes, de ce désir de ségrégation. Ainsi des baux de location/vente (baux à rente) contiennent parfois une clause qui impose que le preneur de la maison n'y loge ni gens de métier sale ou trop bruyant, ni femmes mal renommées ou scandaleuses.

29. Paris est un sujet qui effraye un peu le chercheur individuel. Trop vaste pour être correctement dominé, mais surtout très inégalement documenté. On sait que le gros des archives municipales a disparu dans l'incendie de l'Hôtel de Ville en 1871. Plus qu'ailleurs l'historien doit donc marier, en séries reconstituées avec plus ou moins de réussite, des sources à la fois abondantes et lacunaires.

30. Je songe par exemple à la thèse que Florence Broutin a commencée à l'université de Paris 8 et qui s'attachera aux Parisiennes vues dans les sources judiciaires du Châtelet. 


\section{RÉSUMÉS}

Les statuts de métiers du XIII ${ }^{\mathrm{e}}$ siècle, la grande Ordonnance de Jean le Bon (1350) et le statut des lingères (1485) jalonnent ce parcours de l'histoire des Parisiennes qui gagnent leur vie dans l'atelier et la boutique à l'époque médiévale. Le travail féminin est reconnu, il doit obéir aux mêmes règles que le travail masculin. Il s'exerce dans quelques métiers uniquement féminins, et dans des métiers où hommes et femmes travaillent ensemble. La recherche ne permet pas encore de dire si, au cours de ces trois siècles, cet espace de liberté par le travail pour les femmes, s'est consolidé, agrandi ou s'il s'est restreint.

Trades statutes in the XIII ${ }^{\text {th }}$ century, the great Ordinance of Jean le Bon and the statute of linenclothing women workers mark out the history of the parisian women who earned living in workshops and in shops. Female work is recognized and obeys the same rules as male work. It is to be found both in female trades and in trades where men and women work together. Present day research is unable to say whether in the course of three centuries the freedom done by women at work was extended or curbed.

\section{AUTEUR}

\section{SIMONE ROUX}

Simone ROUX est professeur d'histoire médiévale. Elle enseigne à l'université de Paris 8 , Vincennes-Saint-Denis. Elle a publié La Maison dans l'histoire, Paris, Albin Michel , 1976 ; La Rive gauche des escholiers(XVe siècle), Paris, Christian, 1992 ; elle a également participé à des ouvrages collectifs : Paris, Genèse d'un paysage, Paris, Picard, 1989 et Histoire de Saint-Denis, Toulouse, Privat, 1988. 